\title{
Rank-defect integer estimation and phase-only modernized GPS ambiguity resolution
}

\author{
P. J. G. Teunissen, D. Odijk \\ Department of Mathematical Geodesy and Positioning, Delft University of Technology, Thijsseweg 11, 2629 JA Delft, The Netherlands \\ e-mail: p.j.g.teunissen@citg.tudelft.nl; Tel.: +31-15-278-2558; Fax: +31-15-278-3711
}

Received: 27 September 2001 / Accepted: 15 April 2002

\begin{abstract}
Carrier-phase ambiguity resolution is usually based on the assumption that the underlying model of observation equations is of full rank. In this contribution the model of observation equations is assumed to be of less than full rank. The well-known three-step procedure of integer least squares is generalized and it is shown how the solution can be affected by the rank deficiency. Although the theory is of interest in its own right, a prime application is found in the problem of phase-only ambiguity resolution in the presence of ionospheric delays. The impact of the third global positioning system (GPS) frequency is therefore studied and it is shown by means of suitable ambiguity transformation which ambiguities are integer estimable and which are not in the case of phase-only modernized GPS. A pitfall when using ionosphere-free linear phase combinations is identified. It is shown that only a particular class of such linear phase combinations permits a parameterization in terms of integer-estimable ambiguities. This pitfall does not manifest itself so clearly with the current dual-frequency GPS system.
\end{abstract}

Keywords: Rank-defect integer least-squares estimation - Phase-only modernized global positioning system - Ambiguity success rate - Ionosphere-free linear combinations

\section{Introduction}

Global Navigation Satellite System (GNSS) ambiguity resolution is the process of resolving the unknown cycle ambiguities of double-difference (DD) carrier-phase data as integers. The sole purpose of ambiguity resolution is to use the integer ambiguity constraints as a means of improving significantly on the precision of the

Correspondence to: P.J.G. Teunissen remaining model parameters, such as baseline coordinates and/or atmospheric delays.

Ambiguity resolution applies to a great variety of current and future GNSS models. These models differ greatly in complexity and diversity. The range from single-baseline models used for kinematic positioning to multi-baseline models used as a tool for studying geodynamic phenomena. The models may or may not have the relative receiver-satellite geometry included. They may also be discriminated as to whether the slave receiver(s) are stationary or in motion. When in motion, we solve for one or more trajectories, since with the receiver-satellite geometry included, we will have new coordinate unknowns for each epoch. We may also discriminate between the models as to whether or not the differential atmospheric delays are included as unknowns. In the case of sufficiently short baselines they are usually excluded. In addition to the current Global Positioning System (GPS) models, carrier-phase ambiguity resolution also applies to the future modernized GPS and the future European Galileo GNSS. An overview of GNSS models, together with their applications in surveying, navigation, geodesy and geophysics, can be found in textbooks such as those of Leick (1995), Parkinson and Spilker (1996), Strang and Borre (1997), Teunissen and Kleusberg (1998), Hofmann-Wellenhof et al. (2001) and Misra and Enge (2001).

The literature on carrier-phase ambiguity resolution is usually based on the assumption that the underlying model of observation equations is of full rank. In this contribution we will assume the model of observation equations to be of less than full rank and show how this affects the ambiguity resolution process. Although the corresponding theory is of interest in its own right, a prime application of the theory is in aiming at phaseonly ambiguity resolution in the presence of ionospheric delays. The absence of pseudorange data and the presence of ionospheric delays will result in a rankdefect model of observation equations. The potential advantage of using carrier-phase-only data is that ambiguity resolution will be freed from the effects of 
pseudorange (or code) multipath. Fast phase-only ambiguity resolution has been shown possible for short baselines (see e.g. Tiberius and de Jonge 1995; Teunissen et al. 1997). For long baselines the situation becomes more problematic due to the presence of ionospheric delays. This is certainly true for the present dual-frequency GPS system. It is therefore of interest to study whether the inclusion of a third frequency, as will be the case with modernized GPS, allows us to improve the performance of phase-only ambiguity resolution significantly. The theory that will be presented is independent of the number of frequencies used and independent of their values. Hence it also applies in principle to the future Galileo system, as well as to its integration with modernized GPS. Similar studies can therefore be performed once the Galileo frequencies are known.

This contribution is organized as follows. In Sect. 2 we present a brief review of the theory of integer ambiguity resolution. In Sect. 3 we consider the problem of rank-defect ambiguity resolution. We generalize the well-known three-step procedure of integer least squares (LS) to account for rank deficiencies in the design matrix of the observation equations. We also discuss the uniqueness of the integer solution. By introducing a suitable ambiguity transformation we are able to identify which ambiguities are integer estimable and which are not. We also show how the real-valued parameters are affected by this transformation. In Sect. 4 we apply the theory to the phase-only modernized GPS model. The rank deficiency is identified and it is shown how it can be eliminated while at the same time retaining the integer nature of the ambiguities. In Sect. 5 we discuss an equivalent formulation of the phase-only full-rank model. It is based on the use of ionosphere-free linear phase combinations. In so doing we point out a pitfall when using ionosphere-free linear phase combinations. It is shown that only a particular class of ionosphere-free linear combinations permits a parameterization in terms of integer-estimable ambiguities. This pitfall does not manifest itself so clearly with the current dual-frequency GPS system. Finally, the results of Sects. 4 and 5 are used in Sect. 6 to compute the phase-only success rates of the integer-estimable ambiguities. This is done for the current dual-frequency GPS and for the future modernized GPS system.

In this contribution we make repeated use of admissible ambiguity transformations. A one-to-one ambiguity transformation is said to be admissible when its transformation matrix is integer and its determinant equal to \pm 1 (i.e. the transformation is volume preserving). Admissible ambiguity transformations are the only one-to-one transformations that preserve the integer nature of the ambiguities (Teunissen 1995a).

The following notation will be used throughout. The $n$-dimensional space of integers is denoted as $Z^{n}$ and the $p$-dimensional space of real numbers as $R^{p}$. The letters $a, z$ and $u$ are reserved to denote integers or integer vectors. The $p$-dimensional vector having only ' 1 's as entries is denoted as $e_{p}$. The matrix which projects orthogonally onto the range space of matrix $A$ is denoted as $P_{A}$. This projector is given as $P_{A}=A\left(A^{T} Q_{y}^{-1} A\right)^{-1}$
$A^{T} Q_{y}^{-1}$, in the case that $A$ is of full rank and the metric is given by the inverse of $Q_{y}$. The projector which projects on the orthogonal complement of the range space of $A$ is denoted as $P_{A}^{\perp}$. In the $n$-dimensional case it is given as $P_{A}^{\perp}=I_{n}-P_{A}$, in which $I_{n}$ denotes the unit matrix of order $n$. The symbol $\otimes$ denotes the Kronecker product of two matrices. The Kronecker product $M \otimes N$ of the $m \times n$ matrix $M$ and the $p \times q$ matrix $N$ is defined as the $m p \times n q$ matrix $M \otimes N=\left(M_{i j} N\right)$, in which matrix $M_{i j} N$ is its $(i, j)$ th partition. Finally, we make a repeated use of the short-hand notation $\|\cdot\|_{M}^{2}=(\cdot)^{T} M^{-1}(\cdot)$ for quadratic forms.

\section{Integer ambiguity resolution}

\subsection{The GNSS model}

As our point of departure we will take the following system of linear(ized) observation equations

$y=A a+B b+e$

where $y$ is the given GNSS data vector of order $m, a$ and $b$ are the unknown parameter vectors respectively of order $n$ and $p$, and $e$ is the noise vector. In principle all the GNSS models can be cast in this frame of observation equations. The data vector $y$ may consist of the 'observed minus computed' single-, dual- or triplefrequency DD phase and/or pseudorange (code) observations accumulated over all observation epochs. The entries of vector $a$ are then the DD carrier-phase ambiguities, expressed in units of cycles rather than range. They are known to be integers, $a \in Z^{n}$. The entries of the vector $b$ will consist of the remaining unknown parameters, such as for instance baseline components (coordinates) and possibly atmospheric delay parameters. They are known to be real-valued, $b \in R^{p}$.

When using the LS principle, the GNSS model can be solved by means of the minimization problem

$\min _{a, b}\|y-A a-B b\|_{Q_{y}}^{2}, \quad a \in Z^{n}, b \in R^{p}$

with $Q_{y}$ the variance-covariance (VC) matrix of the GNSS observables. This LS formulation of the problem of ambiguity resolution was first introduced and coined 'integer least-squares' in Teunissen (1993). It is a nonstandard LS problem due to the integer constraints $a \in Z^{n}$.

The solution of the integer LS problem of Eq. (2) can be obtained in three steps.

$$
\begin{aligned}
& {\left[\begin{array}{l}
\hat{a} \\
\hat{b}
\end{array}\right], \quad\left[\begin{array}{cc}
Q_{\hat{a}} & Q_{\hat{a} \hat{b}} \\
Q_{\hat{b} \hat{a}} & Q_{\hat{b}}
\end{array}\right]} \\
& \check{a}=\arg \min _{a \in Z^{n}}\|\hat{a}-a\|_{Q_{\hat{a}}}^{2} \\
& \check{b}=\hat{b}-Q_{\hat{b} \hat{a}} Q_{\hat{a}}^{-1}(\hat{a}-\check{a})
\end{aligned}
$$

In the first step we simply discard the integer constraints $a \in Z^{n}$ on the ambiguities and perform a standard 
adjustment. As a result we obtain the (real-valued) LS estimates $\hat{a}$ and $\hat{b}$, together with their VC matrix. The solution of this first step is referred to as the 'float' solution. In the second step the 'float' ambiguity estimate $\hat{a}$ is used to compute the corresponding integer LS ambiguity estimate $\check{a}$. Once these integer ambiguities are computed, they are used in the third step to finally correct the 'float' estimate of $b$, so as to obtain the 'fixed' estimate $\check{b}$.

That $\check{a}$ and $\check{b}$ do indeed solve Eq. (2) can be seen as follows. From the orthogonal decomposition

$$
\|y-A a-B b\|_{Q_{y}}^{2}=\|\hat{e}\|_{Q_{y}}^{2}+\|\hat{a}-a\|_{Q_{\hat{a}}}^{2}+\|\hat{b}(a)-b\|_{Q_{\hat{b} \mid \hat{a}}}^{2}
$$

with $\hat{e}=y-A \hat{a}-B \hat{b}$ and $\hat{b}(a)=\hat{b}-Q_{\hat{b} \hat{a}} Q_{\hat{a}}^{-1}(\hat{a}-a)$, it follows that the sought-for minimum is obtained when the second term on the right-hand side is minimized for $a \in Z^{n}$ and the last term is set to zero.

Although we will refrain from discussing the computational intricacies of integer LS estimation, we note that the computation of $\check{a}$ is the most demanding. An efficient mechanization of the integer LS procedure is provided by the LAMBDA (Least-squares AMBiguity Decorrelation Adjustment) method. For more information on the LAMBDA method, we refer to e.g. Teunissen (1993, 1995b) and de Jonge and Tiberius (1996a) or to the textbooks of Strang and Borre (1997), Teunissen and Kleusberg (1998), Hofmann-Wellenhof et al. (2001) and Misra and Enge (2001). Practical results obtained with it can be found in, for example, Han (1995), Tiberius and de Jonge (1995), de Jonge and Tiberius (1996b), de Jonge et al. (1996), Boon and Ambrosius (1997), Boon et al. (1997), Tiberius et al. (1997), Jonkman (1998), Cox and Brading (1999) and Peng et al. (1999).

\subsection{The ambiguity probability mass function}

In order to evaluate the expected performance of ambiguity resolution, we need the probability mass function (PMF) of the estimated ambiguities $\breve{a}$. If we denote the probability density function (PDF) of $\check{a}$ as $p_{\hat{a}}(x)$, the PMF of $\check{a}$ follows as

$P(\check{a}=z)=\int_{s_{z}} p_{\hat{a}}(x) \mathrm{d} x, \quad z \in Z^{n}$

with the pull-in regions $S_{z}=\left\{x \in R^{n} \mid\|x-z\|_{Q_{\hat{a}}}^{2} \leq\right.$ $\left.\|x-u\|_{Q_{\hat{a}}}^{2}, \forall u \in Z^{n}\right\}$ as the regions of integration. The above expression holds for any distribution the 'float' ambiguities $\hat{a}$ might have. In most GNSS applications, however, we assume the vector of observables $y$ to be normally distributed. The estimator $\hat{a}$ is then normally distributed too, with mean $a \in Z^{n}$ and VC matrix $Q_{\hat{a}}$. Its probability density function therefore reads

$p_{\hat{a}}(x)=\frac{1}{\sqrt{\operatorname{det}\left(Q_{\hat{a}}\right)}(2 \pi)^{\frac{1}{2} n}} \exp \left\{-\frac{1}{2}\|x-a\|_{Q_{\hat{a}}}^{2}\right\}$
Of the above PMF, the probability of correct integer ambiguity estimation, $P(\check{a}=a)$, is of particular interest. It describes the expected success rate of GNSS ambiguity resolution. In Teunissen (1999a) it is shown that the integer LS estimator is optimal in the sense that it has the largest success rate of all admissible integer estimators. Different ways of evaluation the LS success rate have been given in Teunissen (1998). In the present contribution we will make use of the very-easy-tocompute lower bound

$\prod_{i=1}^{n}\left(2 \Phi\left(\frac{1}{2 \sigma_{i \mid I}}\right)-1\right) \leq P(\check{a}=a)$

with

$\Phi(x)=\int_{-\infty}^{x} \frac{1}{\sqrt{2 \pi}} \exp \left\{-\frac{1}{2} v^{2}\right\} \mathrm{d} v$

and where the $\sigma_{i \mid I}, i=1, \ldots, n$, denote the sequential conditional standard deviations of the decorrelated ambiguities. This lower bound was introduced in Teunissen (1999a) and it is presently the sharpest lower bound available for Eq. (7); see e.g. Thomsen (2000).

\subsection{The 'fixed' baseline's probability of concentration}

Ambiguity resolution is not a goal in itself. The sole purpose of ambiguity resolution is to be able to improve the quality of the solution of the remaining real-valued model parameters. In order to describe the quality of the 'fixed' solution, we would like to know how close we can expect the estimate $\check{b}$ to be to the unknown but true value $b$. As a measure of confidence, we take

$P(\check{b} \in \Omega)=\int_{\Omega} p_{\breve{b}}(x) \mathrm{d} x \quad$ with $\Omega \subset R^{p}$

in which $p_{\breve{b}}(x)$ denotes the PDF of $\breve{b}$. However, in order to evaluate this integral, we first need to make a choice about the shape and location of the subset $\Omega$. Since it is common practice in GNSS positioning to use the VC matrix of the conditional estimator $\hat{b}(a)$ as a measure of precision for the 'fixed' solution, the VC matrix $Q_{\hat{b} \mid \hat{a}}$ will be used to define the shape of the confidence region. For its location, we choose the confidence region to be centered at $b$. With these choices of shape and location, the region $\Omega$ takes the form

$\Omega=\left\{x \in R^{p} \mid(x-b)^{T} Q_{\hat{b} \mid \hat{a}}^{-1}(x-b) \leq \beta^{2}\right\}$

The size of the region can be varied by varying $\beta$.

In Teunissen (1999b) it is shown that the integral of Eq. (8) can be evaluated as the following weighted probability sum of non-central Chi-square distributions:

$P(\check{b} \in \Omega)=\sum_{z \in Z^{n}} P\left(\chi^{2}\left(p, \lambda_{z}\right) \leq \beta^{2}\right) P(\check{a}=z)$ 
with

$\lambda_{z}=\left\|\nabla \check{b}_{z}\right\|_{\left.Q_{\hat{b}}\right|_{\hat{a}}}^{2} \quad$ and $\quad \nabla \check{b}_{z}=Q_{\hat{b} \hat{a}} Q_{\hat{a}}^{-1}(z-a)$

and where $\chi^{2}\left(p, \lambda_{z}\right)$ denotes the non-central Chi-square distribution with $p$ degrees of freedom and non-centrality parameter $\lambda_{z}$.

This result shows that the probability of $\breve{b}$ lying inside the ellipsoidal region $\Omega$ centered at $b$ equals an infinite sum of probability products. If we consider the two probabilities of these products separately, two effects are observed: first the probabilistic effect of shifting the conditional estimator away from $b$ and second the probabilistic effect of the peakedness or non-peakedness of the ambiguity PMF. The second effect is related to the expected performance of ambiguity resolution, while the first effect has to do with the sensitivity of the conditional estimator to changes in the values of the integer ambiguities. This effect is measured by the non-centrality parameter $\lambda_{z}$. Since the tail of a non-central Chi-square distribution becomes heavier when the noncentrality parameter increases, while the degrees of freedom remain fixed, $P\left(\chi^{2}\left(p, \lambda_{z}\right) \leq \beta^{2}\right)$ gets smaller when $\lambda_{z}$ gets larger.

The two probabilities in the product reach their maximum values when $z=a$. These two maxima can be used to lower bound and to upper bound the probability $P(\check{b} \in \Omega)$ as follows:

$P(\hat{b}(a) \in \Omega) P(\check{a}=a) \leq P(\check{b} \in \Omega) \leq P(\hat{b}(a) \in \Omega)$

with

$P(\hat{b}(a) \in \Omega)=P\left(\chi^{2}(p, 0) \leq \beta^{2}\right)$

These bounds are of practical importance, since it is generally difficult to evaluate Eq. (10) exactly. Note that the two bounds relate the probability of the 'fixed' estimator $\check{b}$ to that of the conditional estimator $\hat{b}(a)$ and to the ambiguity success rate $P(\check{a}=a)$. The above bounds become tight when the success rate approaches 1 . This shows that, although the probability of the conditional estimator always overestimates the probability of the 'fixed' estimator, the two probabilities are close for large values of the success rate. This implies that, in the case of GNSS ambiguity resolution, we should first evaluate the success rate $P(\check{a}=a)$ and make sure that its value is close enough to 1 , before making any inferences on the basis of the distribution of the conditional estimator.

\section{Rank-defect ambiguity resolution}

\subsection{The generalized three-step procedure}

In the previous section it was implicitly assumed that the system of observation equations [Eqs. (1)] is of full rank. Modifications to the procedure of ambiguity resolution are in order if this is not the case. In this section we will show which changes are necessary if the design matrix $(A, B)$ is not of full rank.
We will first reformulate the orthogonal decomposition of Eq. (4) such that the full-rank assumption of matrix $(A, B)$ is not necessary. We have

$$
\begin{aligned}
\| y- & A a-B b \|_{Q_{y}}^{2} \\
= & \left\|P_{(A, B)}^{\perp} y\right\|_{Q_{y}}^{2}+\left\|P_{(A, B)}(y-A a-B b)\right\|_{Q_{y}}^{2} \\
= & \|\hat{e}\|_{Q_{y}}^{2}+\left\|P_{(A, B)}(A(\hat{a}-a)+B(\hat{b}-b))\right\|_{Q_{y}}^{2} \\
= & \|\hat{e}\|_{Q_{y}}^{2}+\left\|P_{P_{B}^{\perp} A} A(\hat{a}-a)\right\|_{Q_{y}}^{2} \\
& +\left\|P_{B}(A(\hat{a}-a)-B(\hat{b}-b))\right\|_{Q_{y}}^{2} \\
= & \|\hat{e}\|_{Q_{y}}^{2}+\left\|P_{B}^{\perp} A(\hat{a}-a)\right\|_{Q_{y}}^{2} \\
& +\left\|P_{B} A(\hat{a}-a)-B(\hat{b}-b)\right\|_{Q_{y}}^{2}
\end{aligned}
$$

In the first equation use has been made of $I_{n}=P_{(A, B)}^{\perp}+P_{(A, B)}$ and the fact that the range spaces of these two projectors are mutually orthogonal. The second equation follows from the definition of the LS residual, $\hat{e}=y-P_{(A, B)} y=P_{(A, B)}^{\perp} y$, and from the definition of the LS parameter solution, $P_{(A, B)} y=A \hat{a}+B \hat{b}$. In the third equation we made use of the projector decomposition $P_{(A, B)}=P_{P_{B}^{\perp} A}+P_{B}$ and the fact that the range spaces of $P_{P_{B}^{\perp} A}$ and $P_{B}$ are mutually orthogonal. The last equation follows since $P_{P_{B}^{\perp} A} A=P_{B}^{\perp} A$ and $P_{B} B=B$

The above decomposition may now be used to generalize the three-step procedure of Eq. (3). First note that $\hat{e}$ is always unique, but that the LS parameter solutions $\hat{a}$ and $\hat{b}$ are generally not unique when $(A, B)$ is rank defect. Such solutions can be obtained by means of the theory of generalized inverses (Bjerhammar 1951, 1973) or by means of the theory of S-transformations (Baarda 1960, 1973). For the connection between generalized inverses and S-transformations, we refer to Teunissen (1985). Once a 'float' solution $\hat{a}$ and $\hat{b}$ have been obtained, we can think of minimizing the sum of the last two squares in the last equation of Eqs. (12). Note that since the equation $B b=B \hat{b}-P_{B} A(\hat{a}-a)$ is consistent for any value of $a$, the last square can always be made equal to zero by using an appropriate choice for $b$. Hence, it is the second square which remains to be minimized as function of $a \in Z^{n}$. The generalized three-step procedure therefore reads

$$
\begin{aligned}
& {\left[\begin{array}{l}
\hat{a} \\
\hat{b}
\end{array}\right]=(A, B)_{\mathrm{LS}}^{-} y} \\
& \check{a}=\arg \min _{a \in Z^{n}}\left\|P_{B}^{\perp} A(\hat{a}-a)\right\|_{Q_{y}}^{2} \\
& \check{b}=B^{-}\left(B \hat{b}-P_{B} A(\hat{a}-\check{a})\right)
\end{aligned}
$$

in which $(A, B)_{\text {LS }}^{-}$denotes an LS inverse of $(A, B)$ and $B^{-}$ denotes a generalized inverse of $B$. The LS inverse may be obtained as $\left((A, B)^{T} Q_{y}^{-1}(A, B)\right)^{-}(A, B)^{T} Q_{y}^{-1}$. Compare Eq. (13) with Eq. (3) and note that they are identical in the case that $(A, B)$ is of full rank. 


\subsection{On the non-uniqueness of ambiguity resolution}

We may now use Eq. (13) to study the uniqueness or non-uniqueness of ambiguity resolution. The 'float' solution - step (1) of Eq. (13) - is clearly not unique. In order to describe the set of all 'float' solutions we first assume that the rank defect of $(A, B)$ equals $r$. The dimension of the null space of $(A, B)$ is then also equal to $r, \operatorname{dim}, N(A, B)=r$. Hence there exists an $(n+p) \times r$ matrix $\left(Z_{1}^{T}, Y_{1}^{T}\right)^{T}$, of which the linearly independent columns satisfy $A Z_{1}+B Y_{1}=0$. Using this span of the null space of $(A, B)$, we may characterize the set of 'float' solutions as

$\left[\begin{array}{l}\hat{a} \\ \hat{b}\end{array}\right]=\left[\begin{array}{l}\hat{a}_{p} \\ \hat{b}_{p}\end{array}\right]+\left[\begin{array}{l}Z_{1} \\ Y_{1}\end{array}\right] x, \quad$ with $x \in R^{r}$

with $\hat{a}_{p}$ and $\hat{b}_{p}$ being a particular solution, obtained for instance by using the generalized LS inverse.

In order to see whether or not the non-uniqueness in the 'float' solution is carried over to the 'fixed' solution, we first consider the integer solution of Eq. (13). The second step of Eq. (13) shows that the possible non-uniqueness in governed by the null space of $P_{B}^{\perp} A$. From pre-multiplying $A Z_{1}+B Y_{1}=0$ by $P_{B}^{\perp}$ we obtain

$P_{B}^{\perp} A Z_{1}=0$

Hence the null space is spanned by the columns of $Z_{1}$. Whether or not the integer solution is unique now depends on the signature of matrix $Z_{1}$. The entries of matrix $Z_{1}$ could be zero, they could be non-zero and non-integer, they could be non-zero but integer, or any combination of the above. The 'float' solution $\hat{a}$ and integer solution $\check{a}$ are both unique when matrix $Z_{1}$ vanishes. In that case the rank defect is concentrated in matrix $B$, as a result of which both $\hat{b}$ and $\breve{b}$ will become non-unique. The vanishing of matrix $Z_{1}$ is, however, not a necessary condition for the integer solution to be unique. The integer solution is also unique when the intersection of the range space of $Z_{1}$ with the space of integers $Z^{r}$ equals 0 . This implies that no non-zero integer vector can be found which lies in the null space of $P_{B}^{\perp} A$. If that happens the integer solution $\check{a}$ will be unique, but the 'float' solution $\hat{a}$ will not. As an example consider the case $n=2, r=1$, with $Z_{1}=(\sqrt{2},-1)^{T}$. No $x \in R$, except $x=0$, can then be found for which $Z_{1} x$ is integer.

For our phase-only study the case where matrix $Z_{1}$ is of full rank $r$, but integer, is of particular interest. The 'float' solution $\hat{a}$ and the integer solution $\check{a}$ will then both be non-unique. The 'float' solution will be non-unique since $Z_{1} \neq 0$ and the integer solution will be non-unique since adding an arbitrary integer vector within the range space of $Z_{1}$ to $a$ would not change the value of the objective function $\left\|P_{B}^{\perp} A(\hat{a}-a)\right\|_{Q_{v}}^{2}$. In order to handle this type of non-uniqueness, we may proceed as follows.

Let the $n \times n$ matrix $Z=\left(Z_{1}, Z_{2}\right)$ be an admissible ambiguity transformation (i.e. it is volume preserving and it has integer entries only) and use the reparametrization $\hat{a}-a=Z(\hat{z}-z)$ to obtain

$$
\left\|P_{B}^{\perp} A(\hat{a}-a)\right\|_{Q_{y}}^{2}=\left\|P_{B}^{\perp} A Z_{2}\left(\hat{z_{2}}-z_{2}\right)\right\|_{Q_{y}}^{2}
$$

Since $P_{B}^{\perp} A Z_{2}$ is of full rank, standard techniques may again be used to minimize the right-hand side of Eq. (16) as function of $z_{2} \in Z^{n-r}$. If we denote the corresponding integer solution as $\check{z}_{2}$, the non-unique but integer LS solution of the original ambiguities becomes

$\check{a}=Z_{1} z_{1}+Z_{2} \check{z}_{2}, \quad$ with $z_{1} \in Z^{r}$

The transformed ambiguities $z_{1}$ are the ones that are not integer estimable. In order to see whether or not the non-uniqueness of the integer ambiguities has an effect on the 'fixed' solution $\breve{b}$, we may extend the above ambiguity reparametrization to a reparametrization of both $a$ and $b$. This gives the one-to-one parameter transformation

$\left[\begin{array}{l}a \\ b\end{array}\right]=\left[\begin{array}{ccc}Z_{1} & Z_{2} & 0 \\ Y_{1} & 0 & I_{p}\end{array}\right]\left[\begin{array}{c}z_{1} \\ z_{2} \\ b^{\prime}\end{array}\right]$

Its inverse reads

$\left[\begin{array}{c}z \\ b^{\prime}\end{array}\right]=\left[\begin{array}{ll}\left(Z_{1}, Z_{2}\right)^{-1} & 0 \\ -\left(Y_{1}, 0\right)\left(Z_{1}, Z_{2}\right)^{-1} & I_{p}\end{array}\right]\left[\begin{array}{l}a \\ b\end{array}\right]$

Hence, the entries $b^{\prime}$ equal their counterparts of $b$ when the corresponding rows of matrix $Y_{1}$ equal zero. These entries of $b^{\prime}$ are therefore not affected by the nonuniqueness of the integer ambiguities. In the next section we will show how to construct the parameter transformation of Eq. (18) for the long-baseline, phase-only GPS model.

\section{Phase-only ambiguity resolution}

\subsection{The phase-only modernized GPS model}

In this section we will consider the long-baseline, phaseonly modernized GPS model. Since the emphasis is on long baselines, the ionospheric delays will be included as unknown parameters in the model. The number of satellites tracked will be denoted as $s+1$. For a single epoch $i(i=1, \ldots, k)$ and a stationary baseline, the triplefrequency linearized system of $3 s$ DD carrier-phase observation equations reads

$\phi_{1}(i)=\lambda_{1} a_{1}-\mu_{1} I(i)+G(i) x$

$\phi_{2}(i)=\lambda_{2} a_{2}-\mu_{2} I(i)+G(i) x$

$\phi_{3}(i)=\lambda_{3} a_{3}-\mu_{3} I(i)+G(i) x$

with $\phi_{j}(i)(j=1,2,3)$ the vector of $s$ DD carrier-phase data on frequency $f_{j}, a_{j}$ the unknown time-invariant vector of the $s$ integer ambiguities of frequency $f_{j}, I(i)$ the vector of the $s$ unknown DD ionospheric delays and $x$ the vector of the three unknown stationary baseline components. The $s \times 3$ matrix $G(i)$ captures the relative receiver-satellite geometry at epoch $i, \lambda_{j}$ (for $j=1,2,3$ ) denote the known wavelengths of modernized GPS and $\mu_{j}$ are equal to $\mu_{1}=1, \mu_{2}=\left(\lambda_{2} / \lambda_{1}\right)^{2}, \mu_{3}=\left(\lambda_{3} / \lambda_{1}\right)^{2}$. 
Since it will be assumed that at least four satellites are tracked, we have $s \geq 3$.

If we collect the single-epoch phase data on the three frequencies together in one vector $\phi(i)=\left(\phi_{1}(i)^{T}, \phi_{2}(i)^{T}\right.$, $\left.\phi_{3}(i)^{T}\right)^{T}$, we may write the system of observation equations in vector-matrix form as

$\phi(i)=\left(\Lambda \otimes I_{m}\right) a-\left(\mu \otimes I_{m}\right) I(i)+\left(e_{3} \otimes G(i)\right) x$

with the diagonal matrix $\Lambda=\operatorname{diag}\left(\lambda_{1}, \lambda_{2}, \lambda_{3}\right)$, $\mu=\left(\mu_{1}, \mu_{2}, \mu_{3}\right)^{T}$ and the vector of ambiguities $a=\left(a_{1}^{T}, a_{2}^{T}, a_{3}^{T}\right)^{T}$. For $k$ epochs, the system of observation equations can be written as

$\phi=\left(e_{k} \otimes\left(\Lambda \otimes I_{m}\right)\right) a-\left(I_{k} \otimes\left(\mu \otimes I_{m}\right)\right) I+G x$

with $\phi=\left(\phi(1)^{T}, \ldots, \phi(k)^{T}\right)^{T}, \quad I=\left(I(1)^{T}, \ldots, I(k)^{T}\right)^{T}$ and $G=\left(\left(e_{3} \otimes G(1)\right)^{T}, \ldots,\left(e_{3} \otimes G(k)\right)^{T}\right)^{T}$. The above system of $3 s k$ observation equations will have a rank defect due to the absence of code data and the presence of the unknown ionospheric delays. The presence of the ionospheric delays requires the use of at least two frequencies, while the absence of the code data requires that at least two epochs of data are used so as to have a change in the relative receiver-satellite geometry. However, even with more than two epochs of data and more frequencies, a rank defect of $s$ will remain. Hence, the redundancy of the triple-frequency phase-only system equals $2 s(k-1)-3$. In the next section we will identify the rank defect and use the theory of the previous sections to identify which of the ambiguities are integer estimable.

\subsection{Ambiguity and baseline estimability}

In order to identify the estimability of the parameters, we first need to find a span of the null space the design matrix. This span is then used to construct an admissible ambiguity transformation, the inverse of which will show which integer ambiguities are estimable and which are not. We will identify the estimability for modernized GPS as well as for the current dual-frequency GPS system.

In the notation of Sect. 2, the design matrices $A$ and $B$ of the triple-frequency system of Eq. (22) read

$A=\underbrace{\left(e_{k} \otimes\left(\Lambda \otimes I_{s}\right)\right)}_{3 s k \times s} \quad$ and $\quad B=\underbrace{\left(-I_{k} \otimes\left(\mu \otimes I_{s}\right), G\right)}_{3 s k \times(s k+3)}$

In the case of dual-frequency GPS, the two design matrices will have the same structure, be it that $\Lambda, \mu$ and $G$ will then have to be replaced by their dual-frequency counterparts. In order to obtain a span $\left(Z_{1}^{T}, Y_{1}^{T}\right)^{T}$ of the null space of $(A, B)$, we need to solve $A Z_{1}+B Y_{1}=0$. The result reads

$Z_{1}=\left(1 \otimes\left(\lambda \otimes I_{s}\right)\right) \quad$ and $\quad Y_{1}=\left[\begin{array}{c}e_{k} \otimes\left(\lambda_{1}^{2} \otimes I_{s}\right) \\ 0\end{array}\right]$ with the wavelength vector equal to either $\lambda=\left(\lambda_{1}, \lambda_{2}, \lambda_{3}\right)^{T}$ or $\lambda=\left(\lambda_{1}, \lambda_{2}\right)^{T}$, depending on whether three or two frequencies are used. This result is easily verified by noting that

$\Lambda \lambda-\mu \lambda_{1}^{2}=0$

However, also note that we cannot yet use the above real-valued matrix $Z_{1}$ to construct an admissible ambiguity transformation $Z=\left(Z_{1}, Z_{2}\right)$. The entries of $Z_{1}$ have to be such that $Z$ becomes integer and volume preserving. In order to show how this can be achieved it is instructive to consider the current dual-frequency GPS system first.

\subsubsection{Dual-frequency GPS}

In the case of dual-frequency GPS it is rather straightforward to construct the sought-for admissible matrix $Z$. In order for $Z_{1}$ to become integer such that an admissible matrix $Z=\left(Z_{1}, Z_{2}\right)$ can be formed, the greatest common divisor of the entries of $Z_{1}$ has to equal 1 . After all, the determinant of $Z$ cannot equal \pm 1 in the case that the greatest common divisor of the integer entries of $Z_{1}$ is larger than 1. A greatest common divisor of 1 can be achieved if we make use of the known wavelength ratio of $L_{2}$ and $L_{1}: \lambda_{2} / \lambda_{1}=77 / 60$. Using this ratio, we can construct a normalized wavelength vector $\bar{\lambda}$ such that its entries have a greatest common divisor equal to 1

$\bar{\lambda}=(60,77)^{T}$

Note that $\bar{\lambda}=\left(60 / \lambda_{1}\right) \lambda$. Since the integer vector $\bar{\lambda}$ is a scaled version of the real-valued vector $\lambda$, replacing $\lambda$ in $Z_{1}$ by $\bar{\lambda}$ will not change the range space of $Z_{1}$. But for $\left(Z_{1}^{T}, Y_{1}^{T}\right)^{T}$ to remain a span of the null space, the same scaling, namely $60 / \lambda_{1}$, has to be applied to $Y_{1}$. With Eq. (26) it is now not difficult to construct a $2 \times 2$ admissible transformation matrix. It reads

$\bar{Z}=\left[\begin{array}{rr}60 & -7 \\ 77 & -9\end{array}\right]$

The sought-for $2 s \times 2 s$ admissible ambiguity transformation matrix therefore reads $Z=\left(\bar{Z} \otimes I_{s}\right)$. From inverting $a=\left(\bar{Z} \otimes I_{S}\right) z$ it becomes clear which ambiguities are integer estimable and which are not. The inversion gives

$z_{1}=9 a_{1}-7 a_{2}$
$z_{2}=77 a_{1}-60 a_{2}$

The $s$ ambiguities of $z_{2}$ are integer estimable, while those of $z_{1}$ are not. In a similar way we find for the reparametrized real-valued parameters $b^{\prime}=b-\left(60 / \lambda_{1}\right)$ $Y_{1} z_{1}$ (i.e. the ionospheric delays and baseline components) that

$I^{\prime}(i)=I(i)-60 \lambda_{1} z_{1}$

$x^{\prime}=x$

This shows that the baseline is not affected by the elimination of the rank defect, but that the ionospheric 
delays are affected. The ionospheric delays become biased due to the presence of the undetermined ambiguities $z_{1}$. However, since the bias is time invariant, the time variability of the ionospheric delays will remain unbiased estimable.

\subsubsection{Modernized GPS}

The identification of the parameter estimability becomes a bit more involved when three frequencies are used. Again we make use of the known wavelength ratios. For modernized GPS they are given as $\lambda_{2} / \lambda_{1}=77 / 60$, $\lambda_{3} / \lambda_{1}=154 / 115$ and $\lambda_{3} / \lambda_{2}=24 / 23$. The normalized wavelength vector having entries of which the greatest common divisor equals 1 is now given as

$\bar{\lambda}=(60 \times 23,77 \times 23,77 \times 24)^{T}$

Note that the scaling has changed to $\bar{\lambda}=((60 \times 23) /$ $\left.\lambda_{1}\right) \lambda$. It is now not immediately obvious how the normalized wavelength vector $\bar{\lambda}$ can be extended to a $3 \times 3$ admissible matrix. In fact, different such matrices exist. If we make use of Hermite's decomposition of an integer matrix (see e.g. Nemhauser and Wolsey 1999), one such matrix is given as

$\bar{Z}=\left[\begin{array}{ccc}60 \times 23 & 1613 & 60 \times 3 \\ 77 \times 23 & 90 \times 23 & 77 \times 3 \\ 77 \times 24 & 90 \times 24 & 241\end{array}\right]$

This matrix is integer, volume preserving $(\operatorname{det} \bar{Z}=1)$ and has $\bar{\lambda}$ as its first column. The sought-for $3 s \times 3 s$ admissible ambiguity transformation therefore reads $Z=\left(\bar{Z} \otimes I_{s}\right)$. From inverting $a=\left(\bar{Z} \otimes I_{s}\right) z$ we find which ambiguities are integer estimable and which are not

$z_{1}=-90 a_{1}+67 a_{2}+3 a_{3}$

$z_{2}=77 a_{1}-60 a_{2}$

$z_{3}=24 a_{2}-23 a_{3}$

The $s$ ambiguities of $z_{1}$ are not integer estimable, while those of $z_{2}$ and $z_{3}$ are. In a similar way we find for the reparametrized real-valued parameters $b^{\prime}=b-\left(\left(60 \times 23 / \lambda_{1}\right)\right) Y_{1} z_{1}$

$I^{\prime}(i)=I(i)-60 \times 23 \lambda_{1} z_{1}$

$x^{\prime}=x$

This shows, as in the dual-frequency case, that the baseline is not affected by the elimination of the rank defect, but that the ionospheric delays are affected. The observation that the baseline remains unbiased estimable is important, since it implies that phase-only ambiguity resolution is possible in principle for long baselines as well.

\subsection{The full-rank model}

Now that we have identified which parameters are estimable and which are not, we can use these results to transform the original rank-deficient system of observation equations to one of full rank.

\subsubsection{Dual-frequency GPS}

In order to reparametrize the original dual-frequency observation equations, we need to consider the first two sets of Eqs. (20). Substitution of $a=\left(\bar{Z} \otimes I_{S}\right) z$, using Eq. (27) and the inverse of Eq. (29), gives

$$
\begin{aligned}
& \phi_{1}(i)=7 \lambda_{1} z_{2}-\mu_{1} I^{\prime}(i)+G(i) x \\
& \phi_{2}(i)=9 \lambda_{2} z_{2}-\mu_{2} I^{\prime}(i)+G(i) x
\end{aligned}
$$

This system will be of full rank in the case that more than a single epoch of data are used. When comparing the above system with the first two sets of Eqs. (20) we note that the $2 s$ ambiguities $a_{1}$ and $a_{2}$ have been replaced by the $s$ transformed ambiguities $z_{2}$ and that the ionospheric delays $I(i)$ have been replaced by $I^{\prime}(i)$. We also note that each dual-frequency pair of DD observation equations will now have one integer ambiguity in common.

\subsubsection{Modernized GPS}

In the case that all three sets of equation of Eq. (20) are taken, substitution of $a=\left(\bar{Z} \otimes I_{S}\right) z$, using Eq. (31) and the inverse of Eq. (33), gives

$\phi_{1}(i)=1613 \lambda_{1} z_{2}+180 \lambda_{1} z_{3}-\mu_{1} I^{\prime}(i)+G(i) x$
$\phi_{2}(i)=2070 \lambda_{2} z_{2}+231 \lambda_{2} z_{3}-\mu_{2} I^{\prime}(i)+G(i) x$
$\phi_{3}(i)=2160 \lambda_{3} z_{2}+241 \lambda_{3} z_{3}-\mu_{3} I^{\prime}(i)+G(i) x$

This system will now be of full rank in the case that more than a single epoch of data are used. When comparing the above system with that of Eqs. (20) we note that the $3 s$ ambiguities $a_{1}, a_{2}$ and $a_{3}$ have been replaced by the $2 s$ transformed ambiguities $z_{2}$ and $z_{3}$, and that the ionospheric delays $I(i)$ have been replaced by $I^{\prime}(i)$. We also note that each DD observation equation now contains two integer ambiguities. The triple-frequency DD observation equations of the same satellite pair will, however, have the same pair of integer ambiguities in common.

The above full rank system of observation equations has been obtained through the construction of the admissible ambiguity transformation of Eq. (31). However, as pointed out earlier, matrix $\bar{Z}$ or Eq. (31) is not the only admissible matrix which has $\bar{\lambda}$ as its first column. Any matrix $U=\bar{Z} V$, with $V$ admissible and structured as

$V=\left[\begin{array}{cc}1 & w^{T} \\ 0 & W\end{array}\right]$

will do the job. This implies that Eqs. (35) is not the only possible full-rank parametrization. When three frequencies are used, a whole range of full-rank parametrizations exist, each dependent on the choice made for matrix $V$. Note that these degrees of freedom are absent in the dual-frequency case. In the dual-frequency case, matrix $V$ is of order two. This implies that $W$ reduces to a scalar equal to \pm 1 . The dual-frequency full-rank 
parametrization of Eq. (34) is therefore unique apart from a possible change in sign.

When using the triple-frequency reparametrization $a=\left(U \otimes I_{S}\right) u$ instead of $a=\left(\bar{Z} \otimes I_{s}\right) z$, some of the parameters and coefficients of the full-rank system will change accordingly. The biased ionospheric delays will then equal $I^{\prime}(i)=I(i)-60 \times 23 \lambda_{1}\left(u_{1}+\left(u_{2}, u_{3}\right)^{T} w\right)$, while the integer-estimable ambiguities will transform according to

$\left[\begin{array}{l}z_{2} \\ z_{3}\end{array}\right]=\left(W \otimes I_{s}\right)\left[\begin{array}{l}u_{2} \\ u_{3}\end{array}\right]$

Note that $W \otimes I_{s}$ is admissible, since $V$ is admissible. An example of a possible choice for $W$ is

$W=\left[\begin{array}{cc}-1 & -26 \\ 9 & 233\end{array}\right]$

Matrix $U$ would then read

$U=\left[\begin{array}{ccc}60 \times 23 & 7 & 2 \\ 77 \times 23 & 9 & 3 \\ 77 \times 24 & 9 & -7\end{array}\right]$

Now that we have been able to reduce the rank-deficient system to one of full rank, standard techniques of ambiguity resolution can again be used to resolve the ambiguities. In Sect. 6 we will discuss the expected performance of such a system.

\section{The equivalent class of virtual short-baseline models}

It may happen that in some applications an explicit solution for the ionospheric delays is not required. In that case there is no need to compute the 'float' solution or the 'fixed' solution of the ionospheric delays. For such cases there are essentially two different approaches which can be used. Either we eliminate the ionospheric delays from the normal equations by using an appropriate reduction of the normal matrix, or we eliminate the ionospheric delays a priori. An a priori elimination amounts to an elimination of the ionospheric delays at the level of the observation equations, i.e. before the normal equations are formed. In this section we will show how we can eliminate the ionospheric delays while retaining the integer nature of the estimable ambiguities. We will start with the dual-frequency case.

\subsection{A virtual single-frequency short-baseline model}

Elimination of the ionospheric delays from Eqs. (34) can be achieved by taking certain linear combinations of the DD carrier-phase data. Since $s$ ionospheric delays need to be eliminated per epoch, only $s$ linearly independent combinations of the $2 s$ carrier phases $\phi(i)=$ $\left(\phi_{1}(i), \phi_{2}(i)\right)^{T}$ can be formed. Let these linear combinations be given as

$\psi(i)=\left(l^{T} \otimes I_{s}\right) \phi(i)$ in which the $2 \times 1$ vector $l$ still needs to be determined. In order to eliminate the ionospheric delays, $l$ has to satisfy $l^{T} \mu=0$. If we want the contribution of the relative receiver-satellite geometry $G(i) x$ to appear in the same way as in Eqs. (34), we also need the condition $l^{T} e_{2}=1$. These two conditions determine $l$ uniquely as

$l=\left(\frac{\lambda_{2} 77}{\lambda_{2} 77-\lambda_{1} 60},-\frac{\lambda_{1} 60}{\lambda_{2} 77-\lambda_{1} 60}\right)^{T}$

If we now apply Eq. (41), with Eq. (40), to Eqs. (34), we obtain the transformed system of $s$ observation equations as

$\psi(i)=\lambda_{\psi} z_{2}+G(i) x \quad$ with $\lambda_{\psi}=\frac{\lambda_{1} \lambda_{2}}{\lambda_{2} 77-\lambda_{1} 60}$

Since the ionospheric delays are absent, the structure of this system resembles that of a single-frequency shortbaseline model with wavelength $\lambda_{\psi}$. In our derivation we have ensured that the two phase-only systems, Eqs. (34) and (42), are equivalent. Hence, both systems will give identical results for the 'float' and 'fixed' solutions of the ambiguities and baseline, provided the proper VC matrix of the observables is used.

\subsection{A virtual dual-frequency short-baseline model}

We will now consider eliminating the ionospheric delays from the triple-frequency system of Eqs. (35). Since $s$ ionospheric delays need to be eliminated per epoch, only $2 s$ linearly independent combinations of the $3 s$ carrier phases can be formed. Let these linear combinations be given as

$\theta_{1}(i)=\left(l_{1}^{T} \otimes I_{S}\right) \phi(i) \quad$ and $\quad \theta_{2}(i)=\left(l_{2}^{T} \otimes I_{S}\right) \phi(i)$

where $l_{1}$ and $l_{2}$ are still to be determined. For Eq. (43) to be independent of the ionospheric delays, both vectors $l_{1}$ and $l_{2}$ will have to be orthogonal to $\mu: l_{1}^{T} \mu=0$, $l_{2}^{T} \mu=0$. These conditions are, however, not sufficient for a unique determination of $l_{1}$ and $l_{2}$. We can therefore add two extra conditions for each of the two vectors. If we want each linear combination to depend on only a single ambiguity, instead of two ambiguities, as is the case with Eqs. (35), the two vectors also need to satisfy $l_{1}^{T} \Lambda \bar{Z}_{3}=0$ and $l_{2}^{T} \Lambda \bar{Z}_{2}=0$, where $\bar{Z}_{2}$ and $\bar{Z}_{3}$ denote the second and the third column of matrix $\bar{Z}$, respectively. If we also want the contribution of the relative receiver-satellite geometry $G(i) x$ to appear in the same way as in Eqs. (35), we also need the conditions $l_{1}^{T} e_{3}=1$ and $l_{2}^{T} e_{3}=1$. This therefore brings the total set of conditions to

$l_{1}^{T} \mu=0, \quad l_{1}^{T} \Lambda \bar{Z}_{3}=0, \quad l_{1}^{T} e_{3}=1$

$l_{2}^{T} \mu=0, \quad l_{2}^{T} \Lambda \bar{Z}_{2}=0, \quad l_{2}^{T} e_{3}=1$

This set of conditions determines the two vectors $l_{1}$ and $l_{2}$ uniquely. The first two conditions of each triple determine the directions of $l_{1}$ and $l_{2}$, respectively, while the last condition determines their length. Note that, 
since $\Lambda \bar{\lambda}$ is parallel to $\mu$, the first condition of each triple can be rephrased as $l_{1}^{T} \Lambda \bar{\lambda}=0$ and $l_{2}^{T} \Lambda \bar{\lambda}=0$, respectively. This, combined with the second condition of each triple, shows that $\Lambda l_{1}$ is orthogonal to the first and the third column of matrix $\bar{Z}$, while $\Lambda l_{2}$ is orthogonal to the first and the second column of $\bar{Z}$. Using Eq. (31), the solution for the two $l$ vectors then follows readily as

$$
\begin{aligned}
& l_{1}=\left(\frac{\lambda_{2} 77}{\lambda_{2} 77-\lambda_{1} 60},-\frac{\lambda_{1} 60}{\lambda_{2} 77-\lambda_{1} 60}, 0\right)^{T} \\
& l_{2}=\left(0, \frac{\lambda_{3} 24}{\lambda_{3} 24-\lambda_{2} 23},-\frac{\lambda_{2} 23}{\lambda_{3} 24-\lambda_{2} 23}\right)^{T}
\end{aligned}
$$

If we now apply Eq. (43), with Eq. (45), to Eqs. (35), we obtain the transformed system of $2 s$ observation equations as

$$
\begin{aligned}
& \theta_{1}(i)=\lambda_{\theta_{1}} z_{2}+G(i) x \quad \text { with } \quad \lambda_{\theta_{1}}=\frac{\lambda_{1} \lambda_{2}}{\lambda_{2} 77-\lambda_{1} 60} \\
& \theta_{2}(i)=\lambda_{\theta_{2}} z_{3}+G(i) x \quad \text { with } \quad \lambda_{\theta_{2}}=\frac{\lambda_{2} \lambda_{3}}{\lambda_{3} 24-\lambda_{2} 23}
\end{aligned}
$$

The structure of this system resembles that of a dualfrequency, short-baseline model with wavelengths $\lambda_{\theta_{1}}$ and $\lambda_{\theta_{2}}$. Since we have ensured in our derivation that the two phase-only systems, Eqs. (35) and (46), are equivalent, both systems will provide identical results for the 'float' and 'fixed' solutions of the ambiguities and baseline, but with Eqs. (46) we have of course to make sure that the proper VC matrix is used for $\theta_{1}(i)$ and $\theta_{2}(i)$. This follows from applying the error propagation law to Eqs. (43).

The above virtual dual-frequency short-baseline model has been obtained from Eqs. (35). However, as noted earlier, Eqs. (35) are only one of the many possible full-rank parametrizations of the triple-frequency phase-only observation equations. In order to obtain the whole class, we need to incorporate the ambiguity transformation of Eq. (37). However, inclusion of this reparametrization into Eqs. (46) will affect the structure of the observation equations. With the reparametrization $z_{2}=w_{11} u_{2}+w_{12} u_{3}, z_{3}=w_{21} u_{2}+$ $w_{22} u_{3}$ the number of ambiguities per observation equation will increase from 1 to 2 again. A structure like Eqs. (46) can be recovered by again taking the appropriate linear combinations. Defining the $2 s$ linear combinations as

$\psi_{1}(i)=\left(m_{1}^{T} \otimes I_{s}\right) \theta(i) \quad$ and $\quad \psi_{2}(i)=\left(m_{2}^{T} \otimes I_{s}\right) \theta(i)$

with $\theta(i)=\left(\theta_{1}(i)^{T}, \theta_{2}(i)^{T}\right)^{T}$, the solution reads

$$
\begin{aligned}
& m_{1}=\left(\frac{\lambda_{\theta_{2}} w_{22}}{\lambda_{\theta_{2}} w_{22}-\lambda_{\theta_{1}} w_{12}},-\frac{\lambda_{\theta_{1}} w_{12}}{\lambda_{\theta_{2}} w_{22}-\lambda_{\theta_{1}} w_{12}}\right)^{T} \\
& m_{2}=\left(\frac{\lambda_{\theta_{2}} w_{21}}{\lambda_{\theta_{2}} w_{21}-\lambda_{\theta_{1}} w_{11}},-\frac{\lambda_{\theta_{1}} w_{11}}{\lambda_{\theta_{2}} w_{21}-\lambda_{\theta_{1}} w_{11}}\right)^{T}
\end{aligned}
$$

If we now apply Eqs. (47), with Eqs. (48), to Eqs. (46), we finally obtain the whole equivalent class of virtual dual-frequency short-baseline models. Its $2 s$ observation equations are given as

$\psi_{1}(i)=\lambda_{\psi_{1}} u_{2}+G(i) x$ with $\lambda_{\psi_{1}}=\frac{\lambda_{\theta_{1}} \lambda_{\theta_{2}}}{\lambda_{\theta_{2}} w_{22}-\lambda_{\theta_{1}} w_{11}}$

$\psi_{2}(i)=\lambda_{\psi_{2}} u_{3}+G(i) x$ with $\lambda_{\psi_{2}}=\frac{\lambda_{\theta_{1}} \lambda_{\theta_{2}}}{\lambda_{\theta_{2}} w_{21}-\lambda_{\theta_{1}} w_{11}}$

Note that the four entries of matrix $W$ can still be chosen freely, provided its admissibility is not provoked. Hence they have to be integer and satisfy $w_{11} w_{22}-w_{21} w_{12}= \pm 1$.

\subsection{A pitfall with ionosphere-free linear combinations}

Linear combinations of the carrier-phase data which are independent of the ionospheric delays are referred to in the GPS literature as ionosphere-free linear phase combinations (see e.g. Hofmann-Wellenhof 2001). In the previous two sections we have seen examples of such ionosphere-free linear phase combinations, namely $\psi(i)$ of Eq. (42) in the dual-frequency case and $\psi_{1}(i)$ or $\psi_{2}(i)$ of Eqs. (49) in the triple-frequency case. These linear combinations were constructed to satisfy the following three conditions.

1. The linear phase combination had to be independent of the ionospheric delays.

2. The linear phase combination's dependence on the relative receiver-satellite geometry had to be identical to that of the original DD carrier phases.

3. The linear phase combination had to depend on a single integer-estimable ambiguity.

These three conditions combined made it possible to construct virtual short-baseline models like Eq. (42) or Eqs. (49). Their observation equations are structured like short-baseline DD carrier-phase equations and they contain ambiguities which are ensured to be integer estimable.

At this point we may wonder whether we could have obtained the same results when applying the above conditions to the original DD observation equations, Eqs. (20), instead of to the full-rank observation equations Eqs. (34) or Eqs. (35). In order to answer this question we will consider the dual-frequency case first. We have seen that there exists only one linear combination in the dual-frequency case that satisfies the above-given conditions. In fact, the first two conditions are already sufficient for its unique determination. This linear combination reads

$l=\left(\frac{\lambda_{2} 77}{\lambda_{2} 77-\lambda_{1} 60},-\frac{\lambda_{1} 60}{\lambda_{2} 77-\lambda_{1} 60}\right)^{T}$

If we now apply this linear combination to the original dual-frequency DD observation equation of Eqs. (20), we obtain

$\psi(i)=\lambda_{\psi}\left(77 a_{1}-60 a_{2}\right)+G(i) x \quad$ with $\lambda_{\psi}=\frac{\lambda_{1} \lambda_{2}}{\lambda_{2} 77-\lambda_{1} 60}$ 
In order to satisfy the third condition, we may now think of lumping the two ambiguities in $77 a_{1}-60 a_{2}$ together into one single ambiguity, say $z_{2}$, the rationale being that since $a_{1}$ and $a_{2}$ are both integer, 77a $-60 a_{2}$ will be integer as well. And indeed, by substituting $z_{2}=77 a_{1}-60 a_{2}$ into Eq. (51) we recover Eq. (42). Recognizing the correctness of this result, we may be inclined to believe that this simple procedure is valid and sound. Unfortunately it is not, as we will show by means of a triple-frequency example.

It is not difficult to verify that the two independent triple-frequency linear combinations

$$
\begin{aligned}
& l_{1}=\left(\frac{\lambda_{2} 77}{\lambda_{2} 77-\lambda_{1} 60},-\frac{\lambda_{1} 60}{\lambda_{2} 77-\lambda_{1} 60}, 0\right)^{T} \\
& l_{2}^{\prime}=\left(\frac{\lambda_{3} 154}{\lambda_{3} 154-\lambda_{1} 115}, 0,-\frac{\lambda_{1} 115}{\lambda_{3} 154-\lambda_{1} 115}\right)^{T}
\end{aligned}
$$

satisfy above first two conditions. If we apply these two linear combinations to the original triple-frequency DD observation equations of Eqs. (20), we obtain

$$
\begin{aligned}
\theta_{1}(i)= & \lambda_{\theta_{1}}\left(77 a_{1}-60 a_{2}\right)+G(i) x \\
& \text { with } \quad \lambda_{\theta_{1}}=\frac{\lambda_{1} \lambda_{2}}{\lambda_{2} 77-\lambda_{1} 60} \\
\theta_{2}^{\prime}(i)= & \lambda_{\theta_{2}^{\prime}}\left(154 a_{1}-115 a_{2}\right)+G(i) x \\
& \text { with } \quad \lambda_{\theta_{2}^{\prime}}=\frac{\lambda_{3} \lambda_{1}}{\lambda_{3} 154-\lambda_{1} 115}
\end{aligned}
$$

To satisfy third condition, we may now again think of lumping the ambiguities together such that a single ambiguity per observation equation is obtained. This lumping amount to the reparametrization

$$
\left[\begin{array}{l}
z_{2}^{\prime} \\
z_{3}^{\prime}
\end{array}\right]=\left(\left[\begin{array}{ccc}
77 & -60 & 0 \\
154 & 0 & -115
\end{array}\right] \otimes I_{s}\right)\left[\begin{array}{l}
a_{1} \\
a_{2} \\
a_{3}
\end{array}\right]
$$

And indeed, when Eq. (54) is substituted into Eqs. (53) we do obtain a set of observation equations which has a structure that resembles that of Eqs. (49). However, a closer inspection of Eqs. (53) will reveal that this solution does not satisfy all of the above-given conditions. The first two conditions are satisfied, but not the third. It is true, with the substitution of Eq. (54), that each observation equation of Eqs. (53) contains only a single ambiguity. However, it is not true that these ambiguities are integer estimable. In order to demonstrate this, we substitute $a=\left(\bar{Z} \otimes I_{S}\right) z$, with $\bar{Z}$ of Eq. (31), into the right-hand side of Eq. (54). This gives

$$
\left[\begin{array}{l}
z_{2}^{\prime} \\
z_{3}^{\prime}
\end{array}\right]=\left(\left[\begin{array}{ll}
1 & 0 \\
2 & 5
\end{array}\right] \otimes I_{s}\right)\left[\begin{array}{l}
z_{2} \\
z_{3}
\end{array}\right]
$$

which clearly shows that the transformation is not admissible. Although the entries of the $2 \times 2$ matrix are integer, the determinant of this matrix does not equal \pm 1 . This implies that $z_{2}^{\prime}$ and $z_{3}^{\prime}$ will be integer whenever $z_{2}$ and $z_{3}$ are, but it does not imply the converse. For instance, when $z_{2}^{\prime}=1$ and $z_{3}^{\prime}=1$, then $z_{2}=1$ and $z_{3}=-0.2$. Hence, the integerness of the ambiguities is not secured with the one-to-one transformation of Eq. (55). The set of observation equations, Eqs. (53), parametrized in $z_{2}^{\prime}$ and $z_{3}^{\prime}$, is therefore not a member of the class of virtual dual-frequency short-baseline models of Eqs. (49). Its solution will therefore not be equal to the solution obtained from solving the triplefrequency full-rank model of Eqs. (35).

Of course, from a naive algorithmic point of view, we can always apply ambiguity resolution and estimate the ambiguities $z_{2}^{\prime}$ and $z_{3}^{\prime}$ as integers. However, when doing so we would not be solving for the correct model. We would then not be solving for a model in which the integerness of the original DD carrier-phase ambiguities is properly accounted for. Hence, we should be aware of the pitfall involved when using ionosphere-free linear combinations for integer ambiguity resolution and never rely on the simple procedure of lumping integer combinations of ambiguities.

This pitfall did not manifest itself with the current dual-frequency GPS system. The reason this simple, but incorrect, procedure produced the correct result in the dual-frequency case is that in this case only a single ionosphere-free linear combination exists which does the job. In the triple-frequency case, however, there exists a multitude of ionosphere-free linear combinations, but only a particular subset of them will guarantee the integer estimability of the ambiguities.

\section{Phase-only ambiguity success rates}

In this section we will discuss, by means of an example, the expected performance of phase-only ambiguity resolution. We use the probability of correct integer estimation, i.e. the ambiguity success rate, as our measure of performance. The sampling interval was taken equal to $10 \mathrm{~s}$ and the standard deviation of the undifferenced carrier-phase data was set at $2 \mathrm{~mm}$. Choosing a higher sampling rate, with a corresponding reduction in time of successful ambiguity resolution, is possible when it can be assured that the data remain free from time correlation. In order to show the dependence of the ambiguity success rate on the number of satellites tracked, we considered respectively 4, 6 and 8 satellites tracked.

The respective phase-only ambiguity success rates are shown in Fig. 1. Figure 1a shows the success rates for the current dual-frequency system and Fig. 1b shows the success rates for the modernized GPS. As Fig. 1 shows, the success rates get larger when the observational period gets larger or when the number of satellites tracked gets larger. This figure also shows a dramatic improvement in the success rate when the third frequency of modernized GPS is included. However, despite this improvement, relatively long observational periods are still needed for the triple-frequency success rates to become large enough. How large the success rate needs to be depends of course on the application at hand. It depends on how much risk we 

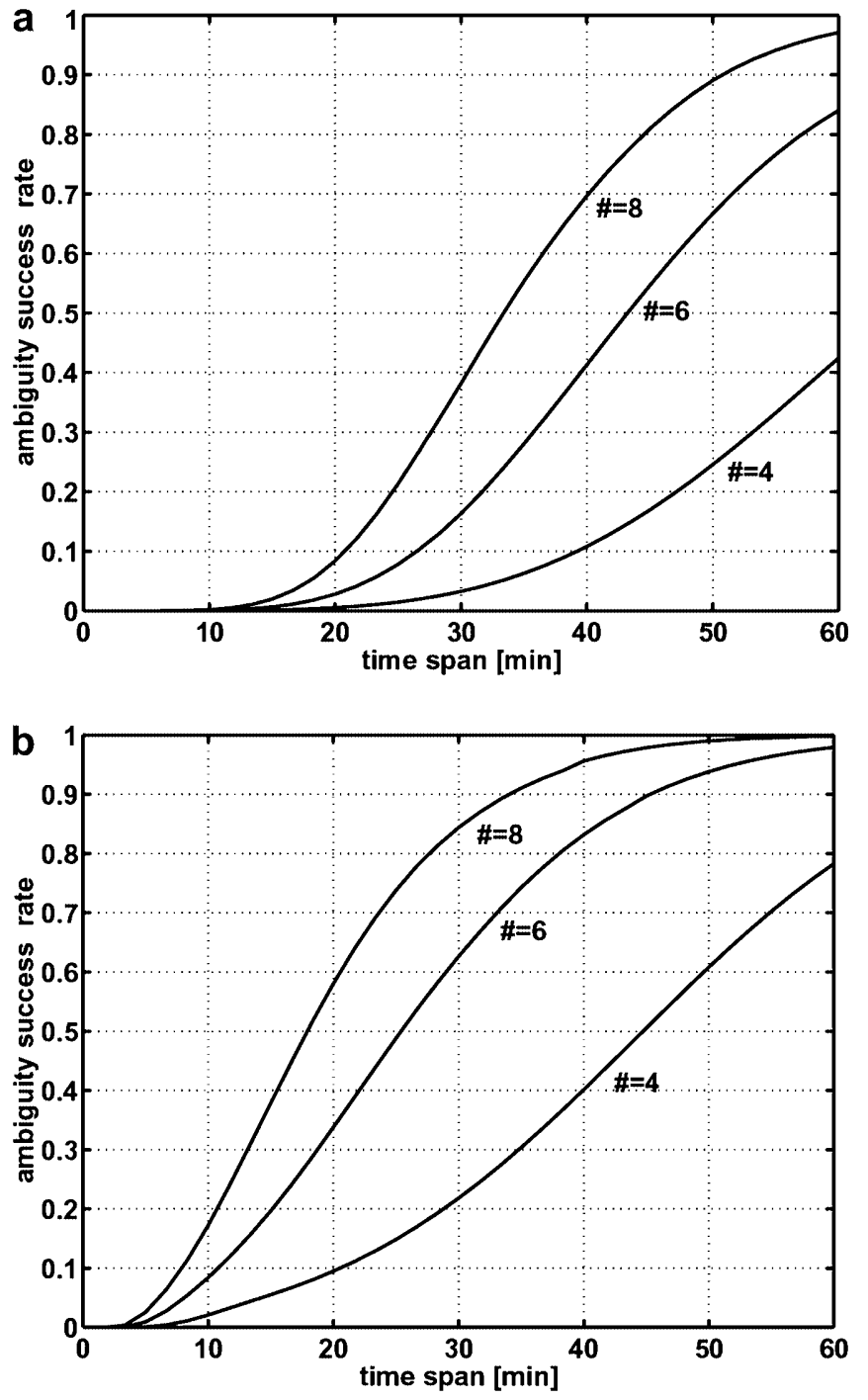

Fig. 1a, b. Phase-only ambiguity success rates as a function of the observation time span and the number of satellites tracked (\#): (a) dual-frequency GPS; (b) modernized GPS

are willing to accept that the wrong ambiguity gets fixed, e.g. 0.1 or $0.01 \%$.

The dual-frequency success rates are extremely poor. This can be understood if we consider the full-rank system of observation equations, Eqs. (34), or its equivalent form, the ionosphere-free system of observation equations, Eq. (42). Since the two vectors $\left(7 \lambda_{1}\right.$, $\left.9 \lambda_{2}\right)^{T}$ and $\left(\mu_{1}, \mu_{2}\right)^{T}$ of Eqs. (34) are almost linear dependent, the precision with which the ambiguities can be estimated will be poor. This poor precision of the 'float' solution translates into a flat PDF and therefore a low probability of correct integer estimation. The same conclusion is reached if we consider Eq. (42). The ionosphere-free linear phase combination $\psi$ has a standard deviation which is about three times larger than that of the original phase data and the virtual wavelength $\lambda_{\psi}$ equals $0.63 \mathrm{~cm}$, which is very small. The relatively low values of the triple-frequency success rate can be explained in a similar way.
Since phase-only, full ambiguity resolution does not seem feasible within relatively short time spans, we might consider partial ambiguity resolution as an alternative. In the case of partial ambiguity resolution we aim at resolving only a subset of the ambiguities, namely the best-determined ambiguities. In our case the best-determined ambiguities are the integer linear combinations $24 a_{2}-23 a_{3}$, i.e. the $z_{3}$ ambiguities of Eqs. (35) and (46), respectively. They correspond with the $L_{2} / L_{3}$ ionosphere-free linear combination $\theta_{2}$. The standard deviation of $\theta_{2}$ is about 17 times the standard deviation of the original phase data and its virtual wavelength $\lambda_{\theta_{2}}$ equals $12.5 \mathrm{~cm}$. The results of partial ambiguity resolution are shown in Fig. 2 and in Table 1. In order to show what can be achieved when the data remain free from time correlation at higher sampling rates, we have included the 1-s sampling rate case as well.
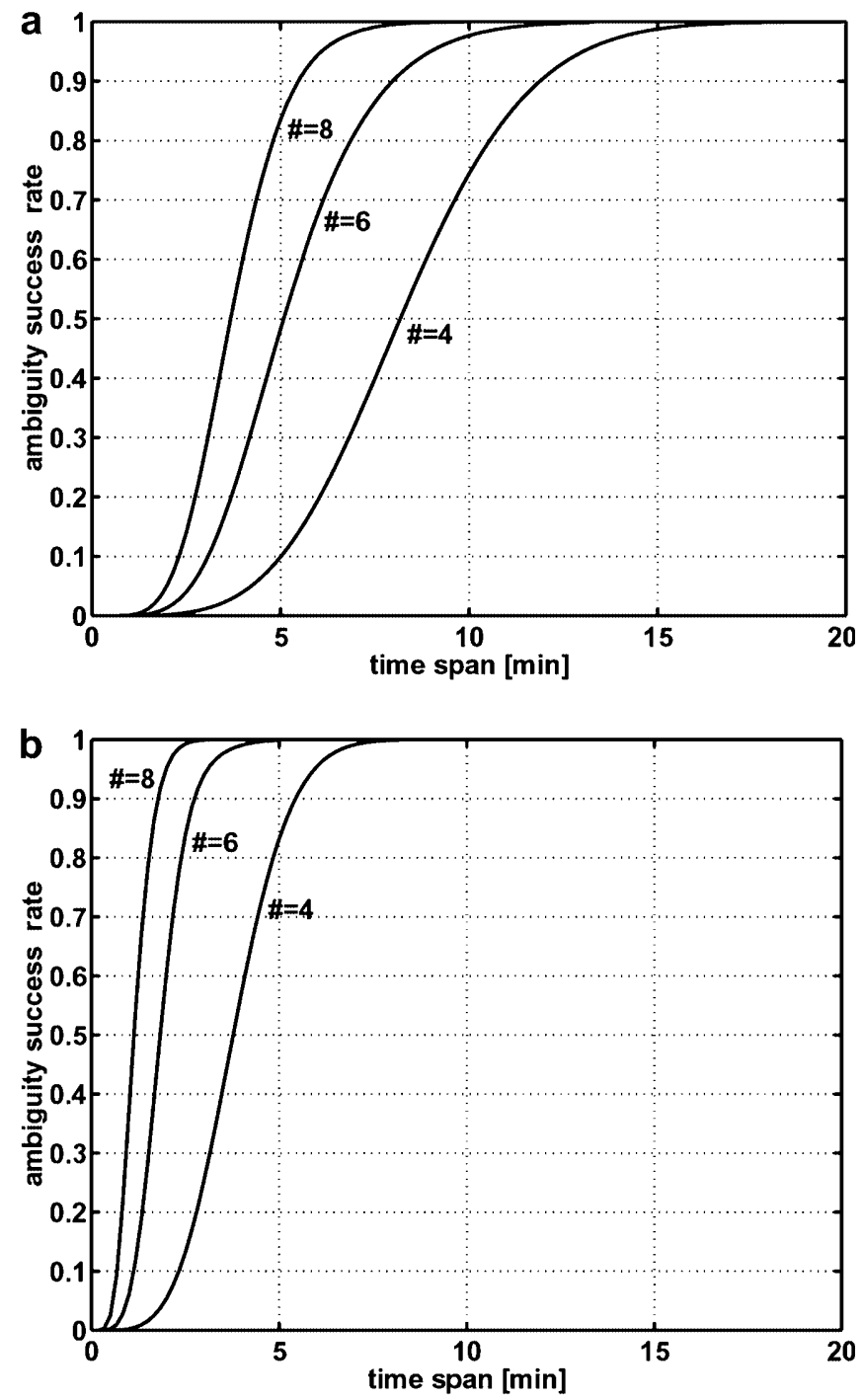

Fig. 2a, b. Phase-only ambiguity success rates of the ambiguities $z_{3}=24 a_{2}-23 a_{3}$ as a function of the observation time span and the number of satellites tracked (\#): (a) 10-s sampling rate; (b) 1-s sampling rate 
Table 1. Baseline precision before and after partial ambiguity resolution

\begin{tabular}{|c|c|c|c|c|}
\hline & $\begin{array}{l}\text { No. satellites } \\
\text { tracked }\end{array}$ & $T(\min )^{\mathrm{a}}$ & Float $(\mathrm{cm})$ & Partial $(\mathrm{cm})$ \\
\hline \multirow[t]{3}{*}{ (a) } & 8 & 9 & 3.5 & 0.4 \\
\hline & 6 & 14 & 2.4 & 0.4 \\
\hline & 4 & 18 & 1.6 & 0.3 \\
\hline \multirow[t]{3}{*}{ (b) } & 8 & 3 & 5.9 & 0.2 \\
\hline & 6 & 5 & 3.2 & 0.2 \\
\hline & 4 & 8 & 1.7 & 0.2 \\
\hline
\end{tabular}

${ }^{a} T$ denotes the observation time span needed to reach an ambiguity success rate of 0.999: (a) 10-s sampling rate; (b) 1-s sampling rate

The partial success rates show a significant improvement as compared to full ambiguity resolution. With a 10-s sampling rate the time for successful ambiguity resolution now lies between about 9 (number of satellites tracked $=8$ ) and 18 (number of satellites tracked $=4$ ) minutes. These numbers are of course still large when compared to what is already achievable with dual-frequency phase-only data over short baselines (see e.g. Teunissen et al. 1997). However, this is understandable and not a fair comparison, since the ionospheric delays are assumed to play a minor role, or no role at all, in the models used for short baselines. Table 1 shows the gain in baseline precision due to partial ambiguity resolution. The gain is largest when eight satellites are tracked, but it gets smaller when fewer satellites are tracked. This is due to the longer time span needed to reach a success rate of 0.999 .

\section{Summary and conclusion}

The literature on carrier-phase ambiguity resolution is usually based on the assumption that the underlying model of observation equations is of full rank. In this contribution we have assumed the model of observation equations to be of less than full rank. This study was in part motivated by the desire to investigate the potential use of phase-only, modernized GPS ambiguity resolution in the presence of ionospheric delays. As a result of the absence of pseudorange data and the presence of ionospheric delays, the corresponding model of observation equations will show a rank defect. tions

Starting from the GPS model of observation equa-

$y=A a+B b+e$

with the rank-defect design matrix $(A, B)$, we first had to generalize the three solution steps of integer LS. The generalized three-step procedure was given as

(2) $\quad \check{a}=\arg \min _{a \in Z^{n}}\left\|P_{B}^{\perp} A(\hat{a}-a)\right\|_{Q_{y}}^{2}$

(3) $\check{b}=B^{-}\left(B \hat{b}-P_{B} A(\hat{a}-\check{a})\right)$
The solutions of the first and the third step are nonunique, while that of the second step is also non-unique if the null space of $P_{B}^{\perp} A$ is spanned by integer vectors. We have shown that this is true if ionospheric delays are present.

By using an appropriate reparametrization, namely

$$
\left[\begin{array}{l}
a \\
b
\end{array}\right]=\left[\begin{array}{ccc}
Z_{1} & Z_{2} & 0 \\
Y_{1} & 0 & I_{p}
\end{array}\right]\left[\begin{array}{l}
z_{1} \\
z_{2} \\
b^{\prime}
\end{array}\right]
$$

we can eliminate the rank defect and obtain a full-rank system of observation equations again. The entries of this one-to-one parameter transformation are chosen such that: (a) the linear-independent columns of $\left(Z_{1}^{T}, Y_{1}^{T}\right)^{T}$ span the null space of $(A, B)$ and (b) matrix $Z=\left(Z_{1}, Z_{2}\right)$ is admissible. The admissibility of matrix $Z$ guarantees that $\left(z_{1}^{T}, z_{2}^{T}\right)^{T}$ is integer whenever $a$ is integer and vice versa. And from the span of the null space it follows that the $z_{2}$ ambiguities are estimable, while the $z_{1}$ ambiguities are not. The resulting full-rank system of observation equations reads $y=A Z_{2} z_{2}+B b^{\prime}$. The phaseonly full-rank system was given for both the dualfrequency and the triple-frequency GPS case. From the solution of the full-rank system, the general set of integer LS solutions of the DD ambiguities follows as

$\check{a}=Z_{1} z_{1}+Z_{2} \arg \min _{z_{2} \in Z^{n-r}}\left\|P_{B}^{\perp} A Z_{2}\left(\hat{z}_{2}-z_{2}\right)\right\|_{Q_{y}}^{2}$

When $s+1$ satellites are tracked, the rank defect of the phase-only system of observation equations equals $s$. This implies that the number of independent integerestimable ambiguities equals $s$ in the dual-frequency case and $2 s$ in the triple-frequency case. As a result it follows that the DD ambiguities are not estimable, only particular integer linear combinations of them. In the dualfrequency GPS case they are given as $z_{2}=77 a_{1}-60 a_{2}$, while in the triple-frequency, modernized GPS case they are given as

$z_{2}=77 a_{1}-60 a_{2}$

$z_{3}=24 a_{2}-23 a_{3}$

These ambiguities are, however, not unique, since any admissible transformation applied to them will again give an independent set of integer-estimable ambiguities. When the LS success rate is used as measure of performance, then all admissible combinations of these integer-estimable ambiguities will perform similarly.

Once the rank defect has been eliminated, it again becomes possible to resolve the ambiguities using standard techniques. However, this only makes sense if the parameters of interest have remained unbiased estimable. This was shown to be true for the baseline components, but false for the ionospheric delays. The latter become biased by an unknown but constant offset. Phase-only ambiguity resolution can be based on the full-rank observation equations explicitly parameterized in the ionospheric delays, or on their equivalent forms in which these delays have been eliminated a priori. We have given both formulations for the dual- 
frequency as well as for the triple-frequency modernized GPS case. In so doing we also identified a pitfall when using ionosphere-free linear phase combinations. It was shown that not all ionosphere-free linear combinations permit a parametrization in terms of integer-estimable ambiguities. This pitfall has until now not been recognized, probably because it does not manifest itself with the current dual-frequency GPS system.

Although phase-only ambiguity resolution is possible in principle, it only makes sense if we can determine the integer-estimable ambiguities reliably using a not-toolong observation time span. We therefore computed, as an example, the success rates of phase-only ambiguity resolution for both the dual-frequency and the triplefrequency, modernized GPS case. For both cases it was shown that phase-only, full ambiguity resolution is not really feasible within relatively short time spans. Partial ambiguity resolution, using the $z_{3}$ ambiguities, was therefore considered as an alternative. The time needed to reach a success rate of 0.999 or larger was found to lie between 9 and 18 minutes when using a 10-s sampling rate, and between 3 and 8 minutes in the case of a 1-s sampling rate.

\section{References}

Baarda W (1960) De betekenis van varianties van coordinaten bij berekening van puntsbepalingsystemen in het platte vlak. Laboratorium voor Geodetische Rekentechniek, Delft

Baarda W (1973) S-transformations and criterion matrices. Publications of geodesy, New series, vol 5, no. 1. Netherlands Geodetic Commission, Delft

Bjerhammar A (1951) Application of calculus of matrices to the method of least-squares with special reference to geodetic calculations. Trans Roy Inst Technol Stockholm, no. 49, 86 pp.

Bjerhammar A (1973) Theory of errors and generalized matrix inverses. Elsevier, Amsterdam

Boon F, Ambrosius B (1997) Results of real-time applications of the LAMBDA method in GPS based aircraft landings. Proc KIS97 Banff, Canada, June 3-6, 1997, pp 339-345

Boon F, de Jonge PJ, Tiberius CCJM (1997) Precise aircraft positioning by fast ambiguity resolution using improved troposphere modelling. Proc ION GPS 97, Kansas City, USA, Sept. 16-19, 1997, pp 1877-1884

Cox DB, Brading JDW (1999) Integration of LAMBDA ambiguity resolution with Kalman filter for relative navigation of spacecraft. Proc ION-NTM 99, San Diego, USA, Jan. 25-27, 1999, pp 739-745

de Jonge PJ, Tiberius CCJM (1996a) The LAMBDA method for integer ambiguity estimation: implementation aspects. LGR series no. 12, Delft Computing Centre, Delft

de Jonge PJ, Tiberius CCJM (1996b) Integer estimation with the LAMBDA method. In: Beutler $G$ et al. (eds) Proc IAG Symposium no. 115, GPS trends in terrestrial, airborne and spaceborne applications. Springer, Berlin Heidelberg New York, pp 280-284

de Jonge PJ, Tiberius CCJM, Teunissen PJG (1996) Computational aspects of the LAMBDA method for GPS ambiguity resolution. Proc ION-GPS 96, Kansas City, USA, Sept. 17-20, 1996, pp 935-944

Han S (1995) Ambiguity resolution techniques using integer leastsquares estimation for rapid static or kinematic positioning. Proc 2nd Symp Satellite Navigation Technology: 1995 and beyond, Brisbane, Australia, April 24-28

Hofmann-Wellenhof B, Lichtenegger H, Collins J (2001) Global positioning system: theory and practice, 5th edn. Springer, Berlin Heidelberg New York

Jonkman NF (1998) Integer GPS ambiguity estimation without the receiver-satellite geometry. LGR series, no. 18, Delft Geodetic Computing Centre, Delft

Leick A (1995) GPS satellite surveying, 2nd edn. John Wiley, New York

Misra P, Enge P (2001) Global positioning system: signals measurement, and performance. Ganga-Jamuna Press, Lincoln

Nemhauser GL, Wolsey LA (1999) Integer and combinational optimization. John Wiley, New York

Parkinson B, Spilker JJ (eds) (1996) GPS: theory and applications, vols 1 and 2. AIAA, Washington, DC

Peng HM, Chang FR, Wang LS (1999) Attitude determination using GPS carrier phase and compass data. Proc ION-NTM 99, San Diego, USA, Jan. 25-27, 1999, pp 727-732

Strang G, Borre K (1997) Linear algebra, geodesy, and GPS. Wellesley-Cambridge Press, Massachusetts

Teunissen PJG (1985) Generalized inverses, adjustment, the datum problem and S-transformations. In: Grafarend E, Sanso F (eds) Optimization and design of geodetic networks. Springer, Berlin Heidelberg New York, pp 11-55

Teunissen PJG (1993) Least-squares estimation of the integer GPS ambiguities. Invited lecture, Sect IV Theory and methodology, IAG General Meeting, Beijing, August. Also in: LGR Series no. 6, Geodetic Computing Centre, Delft

Teunissen PJG (1995a) The invertible GPS ambiguity transformations. Manuscr Geod 20: 489-497

Teunissen PJG (1995b) The least-squares ambiguity decorrelation adjustment: a method for fast GPS integer ambiguity estimation. J Geod 70: 65-82

Teunissen PJG (1998) On the integer normal distribution of the GPS ambiguities. Artific Sat 33(2): 49-64

Teunissen PJG (1999a) An optimality property of the integer leastsquares estimator. J Geod 73: 587-593

Teunissen PJG (1999b) The probability distribution of the GPS baseline for a class of integer ambiguity estimators. J Geod 73: 275-284

Teunissen PJG, Kleusberg A (eds) (1998) GPS for geodesy, 2nd edn. Springer, Berlin Heidelberg New York

Teunissen PJG, Tiberius CCJM, de Jonge PJ (1997) The leastsquares ambiguity decorrelation adjustment: its performance on short GPS baselines and short observation spans. J Geod 71: $589-602$

Thomsen HF (2000) Evaluation of upper and lower bounds on the success probability. Proc ION GPS-2000, Salt Lake City, USA, Sept. 19-22, 2000, pp 183-188

Tiberius CCJM, de Jonge PJ (1995) Fast positioning using the LAMBDA method. Proc DSNS-95, Bergen, Norway, April 2428, 1995, paper 30 